Artigo Original

\title{
Adesão de mulheres mastectomizadas ao início precoce de um programa de reabilitação*
}

\author{
Adherence to an early rehabilitation program among women who underwent mastectomy \\ Adhesión de mujeres mastectomizadas al inicio de un programa de rehabilitación temprana

\begin{abstract}
Maria Gaby Rivero de Gutiérrez ${ }^{1}$, Michelle Manzoli Bravo², Daniella Cristina Chanes $^{3}$, Maria Claudia Rodrigues De Vivo ${ }^{4}$, Gabriela Olbrich de Souza ${ }^{5}$
\end{abstract}

\section{RESUMO}

Objetivos: Verificar a adesão de mulheres mastectomizadas ao início precoce de um programa de reabilitação da amplitude de movimento do ombro homolateral à cirurgia e identificar as dificuldades na realização dos exercícios e das atividades de vida diária. Métodos: Estudo prospectivo desenvolvido no Ambulatório de Oncomastologia do Hospital São Paulo/Universidade Federal de São Paulo, no período de 2003 a 2004. A amostra constitui-se de 28 mulheres mastectomizadas que aceitaram participar do programa de reabilitação, desde a alta hospitalar até o primeiro retorno ambulatorial. Resultados: Aderiram ao programa $64,2 \%$ das pacientes e $82,1 \%$ referiram dificuldade para execução dos exercícios, principalmente devido à dor. A maioria realizou as atividades de vida diária sem dificuldades. Conclusão: Faz-se necessário um melhor controle da dor pós-operatória e reforço das orientações para incrementar a adesão das pacientes ao programa de reabilitação.

Descritores: Cooperação do paciente; Neoplasias mamárias; Mastectomia; Enfermagem em reabilitação; Enfermagem oncológica

\section{ABSTRACT}

Objectives: The main objective was to measure adherence to an early rehabilitation program among women who underwent mastectomy. Other specific objectives included the identification of women's difficulties to comply with the exercise prescription and to perform daily life activities, and whether women's adherence to the rehabilitation program was effective in regaining their shoulder's full range of motion on the side of the surgery. Methods: A prospective study design was used. A sample of 28 women who underwent mastectomy and agreed to participate in an early rehabilitation program from discharge to the first outpatient clinic follow-up participated in the study. Data were collected from 2003 to 2004 in the oncomastology outpatient clinic of the Hospital São Paulo of the University Federal of São Paulo. Results: The majority of women $(63.2 \%)$ adhered to the early rehabilitation program. The majority of women (82.1\%) also reported having difficulties to perform the prescribed exercise program due to pain but not with daily life activities. Conclusion: Pain management and patient education must be addressed to improve adherence to the early rehabilitation program.

keywords: Patient compliance; Breast neoplasm; Mastectomy; Rehabilitation nursing, Oncologic nursing

\section{RESUMEN}

Objetivos: Verificar la adhesión de mujeres mastectomizadas al inicio de un programa de rehabilitación temprana de la amplitud de movimiento del hombro homolateral a la cirugía, e identificar las dificultades en la realización de los ejercicios y de las actividades de vida diaria. Métodos: Estudio prospectivo desarrollado en consulta externa de Oncomastología del Hospital São Paulo/Universidade Federal de São Paulo, en el período 2003 al 2004. La muestra se constituyó de 28 mujeres mastectomizadas que aceptaron participar del programa de rehabilitación, desde el alta hospitalario hasta el primer retorno ambulatorio. Resultados: La adhesion al programa fue del 64,2\% de las pacientes y el $82,1 \%$ refirieron dificultad para la ejecución de los ejercicios, principalmente debido al dolor. La mayoría realizó las actividades de vida diaria sin dificultades. Conclusión: Se hace necesario un mejor control del dolor post operatorio y refuerzo de las orientaciones para incrementar la adhesión de las pacientes al programa de rehabilitación.

Descriptores: Cooperación del paciente; Neoplasias mamarias; Mastectomía; Enfermería en rehabilitación; Enfermería oncológica

\footnotetext{
* Trabalho realizado na Escola de Enfermagem da Universidade Federal de São Paulo e apresentado no IX Colóquio Panamericano de Ivestigación en Enfermería. Lima, Peru. 2004.

${ }^{1}$ Professora Adjunto do Departamento de Enfermagem - Universidade Federal de São Paulo -UNIFESP.- São Paulo (SP) Brasil.

${ }^{2}$ Acadêmica em Enfermagem e bolsistas de Iniciação Cientifica CNPq. Universidade Federal de São Paulo- UNIFESP-São Paulo (SP) - Brasil.

${ }^{3}$ Acadêmica em Enfermagem e bolsistas de Iniciação Cientifica CNPq. Universidade Federal de São Paulo- UNIFESP- São Paulo (SP) - Brasil.

${ }^{4}$ Acadêmica em Enfermagem e bolsistas de Iniciação Cientifica CNPq. Universidade Federal de São Paulo- UNIFESP- São Paulo (SP) - Brasil.

${ }^{5}$ Fisioterapeuta, Pós-graduanda da Disciplina de Uroginecologia do Departamento de Ginecologia - Universidade Federal de São Paulo- UNIFESP-São Paulo (SP) - Brasil.
}

Autor Correspondente: Maria Gaby Rivero de Gutiérrez

R. Napoleão de Barros, 754 - Vila Clementino - São Paulo - SP

Artigo recebido em 26/06/2006 e aprovado em 24/03/2007

CEP. 04024-002 E-mail: mggutierrez@denf.epm.br 


\section{INTRODUÇÃO}

A reabilitação física precoce de pacientes mastectomizadas tem como meta principal prevenir complicações que possam limitar a amplitude dos movimentos do membro superior homolateral à cirurgia.

Estudos comprovam que a imobilização total do membro homolateral à cirurgia, antigamente preconizada por muitos cirurgiões visando preservar a aderência das bordas cirúrgicas, é a grande responsável pelo aparecimento de fibroses, retrações e aderências, limitando a amplitude dos movimentos e aumentando o risco de linfedema e outras complicações. Essas complicações podem ser evitadas com a adoção de um programa de exercícios de reabilitação funcional iniciado o mais precocemente possível ${ }^{(1-4)}$. Os benefícios dessa prática estendem-se para além da área física, obtendo-se resultados favoráveis, também na área psicológica ${ }^{(2-3,5-6)}$.

Para que a reabilitação alcance os resultados esperados, além de ter inicio imediato, deve contar com a adesão das pacientes. Aderir ao tratamento significa concordar com a proposta terapêutica e seguí-la conforme recomendado $^{(7)}$. A Organização Mundial da Saúde considera a adesão um importante indicador da efetividade do sistema de saúde e ressalta que a qualidade do relacionamento entre a equipe de saúde e o paciente é um dos fatores determinantes da $\operatorname{adesão}^{(8)}$.

Vários fatores influenciam a adesão ao tratamento, dentre eles a característica da terapia, aspectos relacionados com a equipe multiprofissional, variáveis socioeconômicas e características do paciente, como idade, sexo, raça, escolaridade, ocupação, estado civil, religião, hábitos de vida, hábitos culturais, além de crenças relacionadas à saúde e particularidades da doença ${ }^{(9-10)}$. Identificar os fatores que dificultam a adesão precoce ao programa de tratamento é uma forma de otimizar o serviço prestado.

Por outro lado, a mudança de comportamento em saúde implica na crença dos indivíduos de que a prática de determinadas ações lhes trará benefícios, ou seja, que serão capazes de evitar danos ou reduzir sua severidade ${ }^{(11)}$.

Diante do exposto, desenvolveu-se este estudo com pacientes submetidas à cirurgia oncológica de mama, com os seguintes objetivos: verificar a taxa de adesão ao início precoce de um programa de exercícios de reabilitação funcional da amplitude de movimento do ombro homolateral à cirurgia, a ser realizado no domicílio, no período compreendido entre o pós-operatório imediato e o primeiro retorno ambulatorial; identificar as dificuldades referidas pelas pacientes para aderir à realização desses exercícios e verificar quais atividades de vida diária foram realizadas pelas pacientes nesse período.

Cabe destacar que os dados relativos à efetividade do programa de exercícios quanto à amplitude de movimento não foram objeto deste estudo.

\section{MÉTODOS}

Estudo prospectivo, realizado no Setor de Oncomastologia do Hospital São Paulo (HSP) da Universidade Federal de São Paulo (UNIFESP), após aprovação do Comitê de Ética em Pesquisa da UNIFESP.

A amostra constitui-se de 28 mulheres com diagnóstico de câncer de mama, submetidas a Mastectomia ou Quadrantectomia, de um grupo de 44 que aceitaram participar do programa em questão, assinando o Termo de Consentimento Livre e Esclarecido. O programa consistia em uma seqüência de cinco exercícios, sendo dois para relaxamento e três para exercitar a flexão e extensão da articulação do ombro, a serem executados do primeiro ao sétimo ou décimo dia de pós-operatório. Cabe destacar que os exercícios selecionados para o programa de reabilitação, bem como sua seqüência, foram definidos com base na literatura e assessoria de uma fisioterapeuta.

As 16 pacientes que não fizeram parte da amostra foram excluídas devido a diversos motivos, dentre os quais se destacam: falta de condições de compreensão das orientações; problemas de ordem sócio-econômica que impossibilitaram participar do programa; faltas nos dias estipulados, e evolução com infecção no sítio cirúrgico.

Os dados foram coletados no período de agosto de 2003 a agosto de 2004, utilizando como instrumento de coleta uma ficha de seguimento do programa em que constavam dados de identificação da paciente, dados clínicos e de avaliação da realização dos exercícios e das atividades de vida diária no domicílio, antes e após a cirurgia.(Anexo)

\section{Coleta de dados}

Os dados foram coletados em duas etapas, sendo a primeira realizada no pré-operatório, com a finalidade de verificar as condições das pacientes para a realização das atividades de vida diária antes da cirurgia, receber orientações sobre os cinco exercícios a serem executados no período aprazado $\left(1^{\circ}\right.$ dia de pós-operatório até o $1^{\circ}$ retorno ambulatorial), demonstrar esses exercícios e solicitar a repetição, pela paciente, a fim de verificar as condições físicas para realização dos mesmos, corrigir possíveis erros e esclarecer suas dúvidas. Ao término da demonstração era entregue o Manual de Orientações para Reabilitação Pós-operatória em Cirurgia de Mama.

$\mathrm{Na}$ segunda etapa, realizada no primeiro retorno ambulatorial após a cirurgia $\left(7^{\circ}\right.$ ou $\left.10^{\circ} \mathrm{dia}\right)$, as pacientes eram entrevistadas com o objetivo de identificar se houve ou não adesão ao programa, os motivos para a nãoadesão e a realização, ou não, das atividades da vida diária. Também era realizada a avaliação física pela 
fisioterapeuta.

\section{RESULTADOS}

\section{Caracterização da amostra}

Das 28 mulheres submetidas à cirurgia que foram orientadas no pré-operatório e monitoradas no primeiro retorno ambulatorial sobre a adesão ao programa de exercícios e realização das atividades da vida diária, 17 foram submetidas a Mastectomia e 11 a Quadrantectomia, das quais 14 tiveram esvaziamento ganglionar axilar. Apenas uma paciente recebeu quimioterapia neoadjuvante e nenhuma delas foi submetida à reconstrução imediata da mama.

No que se refere à idade, a média encontrada foi de 54,7 anos (mínima 34 e máxima 80), sendo que a faixa etária predominante foi a de 40 a 59 anos (15 pacientes $53,5 \%$ ). Quanto ao grau de escolaridade, predominou o grupo de pacientes que havia cursado somente o Ensino Fundamental $(18-64,2 \%)$.

\section{Realização dos exercícios}

A avaliação realizada mostrou que a maioria das pacientes $18(64,2 \%)$ aderiu ao programa de exercícios de reabilitação. Consideraram-se aderentes ao programa, as mulheres que referiram ter realizado os exercícios com a regularidade recomendada, ou seja, diariamente e três vezes ao dia, bem como aquelas que embora os tivessem realizado diariamente, o fizeram com menor freqüência diária.

A não adesão ao programa foi verificada em 10 pacientes $(35,8 \%)$. Nesta categoria estão incluídas as pacientes que não realizaram os exercícios e as que os realizaram de forma irregular.

Pode-se constatar, ainda, que a maioria das pacientes, $23(82,1 \%)$ referiu ter apresentado dificuldades para aderir ao programa e, as 10 pacientes $(35,8 \%)$ que não aderiram, também apontaram a ocorrência de dificuldades como sendo a causa da não adesão. Esses dados estão apresentados na Tabela 1.

Tabela 1 - Mulheres submetidas à cirurgia por câncer de mama, segundo adesão ao programa de exercícios e dificuldades para sua execução. São Paulo, 2004.

\begin{tabular}{lcccccc}
\hline \multirow{2}{*}{ Itens } & \multicolumn{2}{c}{ Sim } & \multicolumn{2}{c}{ Não } & \multicolumn{2}{c}{ Total } \\
& $\mathbf{N}$ & $\mathbf{\%}$ & $\mathbf{N}$ & $\mathbf{\%}$ & $\mathbf{N}$ & $\mathbf{\%}$ \\
\hline Adesão & 18 & 64,2 & 10 & 35,8 & 28 & 100 \\
Dificuldades & 23 & 82,1 & 5 & 17,9 & 28 & 100 \\
\hline
\end{tabular}

Na Tabela 2, pode-se observar que entre os motivos que dificultaram ou impediram a adesão ao programa, a dor ao realizar os exercícios ou ao tentar realizá-los (10 pacientes), assim como o medo de que sua execução pudesse afetar a operação (nove pacientes), foram apontados com maior freqüência por quem não aderiu ao programa ou por quem realizou os exercícios de forma irregular.

Tabela 2 - Motivos que dificultaram ou impediram a adesão ao programa de exercícios, referidos por mulheres submetidas à cirurgia por câncer de mama. São Paulo, 2004.

\begin{tabular}{lccc} 
& \multicolumn{2}{c}{$\mathrm{n}=23$} \\
\cline { 2 - 3 } Motivos & \multicolumn{2}{c}{ Adesão } & Total \\
\cline { 2 - 3 } & Sim & Não & \\
\hline Medo de afetar a operação & 4 & 6 & 10 \\
Medo de sentir dor & 2 & 2 & 4 \\
Falta de ânimo para realizar os & 1 & 1 & 2 \\
$\begin{array}{l}\text { exercícios } \\
\text { Dor ao realizar os exercícios }\end{array}$ & 7 & - & 7 \\
Dor ao tentar realizar os & - & 5 & 5 \\
exercícios & & & \\
Outros & 7 & 5 & 12 \\
\hline
\end{tabular}

Ressalta-se, ainda, que das quatro pacientes que referiram medo de sentir dor ao realizar os exercícios, duas o classificaram como o motivo responsável por sua não adesão. Apenas duas pacientes mencionaram falta de ânimo para a realização dos mesmos.

$\mathrm{Na}$ categoria "Outros", os motivos apresentados como limitantes para a realização dos exercícios foram: a sensação de "ardência" (quatro pacientes) e "repuxo" (seis pacientes) na parte interna do braço homolateral à cirurgia e na região torácica na qual a mama foi retirada (duas pacientes), sensação de peso no braço operado (uma paciente), fraqueza e mal estar (três pacientes) e falta de sensibilidade na região ao redor da cicatriz operatória e nas mãos (uma paciente).

Tabela 3 - Mulheres submetidas à cirurgia por câncer de mama segundo a realização de atividades de vida diária e grau de dificuldade apresentado. São Paulo, 2004

$\mathrm{n}=28$

\begin{tabular}{|c|c|c|c|c|c|}
\hline \multirow{2}{*}{ Atividades } & \multicolumn{4}{|c|}{ Grau de dificuldade } & \multirow[t]{2}{*}{ Total } \\
\hline & SD & PD & $+/-$ & NC & \\
\hline Tomar banho & 21 & 6 & 1 & - & 28 \\
\hline $\begin{array}{l}\text { Lavar parte superior do } \\
\text { ombro oposto à cirurgia }\end{array}$ & 20 & 6 & 1 & 1 & 28 \\
\hline Escovar os dentes & 28 & - & - & - & 28 \\
\hline $\begin{array}{l}\text { Pentear e/ou prender os } \\
\text { cabelos }\end{array}$ & 22 & 4 & 2 & - & 28 \\
\hline Vestir-se e despir-se & 18 & 9 & 1 & - & 28 \\
\hline Alimentar-se sozinha & 26 & 2 & - & - & 28 \\
\hline
\end{tabular}

$\overline{\mathrm{SD}}$, sem dificuldade; $\mathrm{PD}$, pouca dificuldade; +/-, sem muita nem pouca dificuldade; $\mathrm{NC}$, não consegue realizar. 


\section{Realização das atividades da vida diária}

Pode-se constatar, na Tabela 3 , que no período estudado, o número de mulheres que referiu ter conseguido realizar as atividades de higiene pessoal sem dificuldades variou de $18(64,3 \%)$ a $28(100,0 \%)$. As atividades de vestir-se e despir-se, tomar banho e lavar a parte superior do ombro oposto à cirurgia foram aquelas em que, com maior freqüência, as pacientes referiram ter apresentado alguma dificuldade.

No que diz respeito à realização de atividades domésticas, a maioria das pacientes, 17 (60,7\%), referiu não tê-las executado no período avaliado.

\section{DISCUSSÃO}

Os resultados obtidos neste estudo mostram que a adesão à realização de exercícios físicos relacionados a um programa de reabilitação pós-operatória por câncer de mama enfrenta uma série de dificuldades, uma vez que esbarra em um conjunto de fatores relacionados à patologia, terapêutica proposta, equipe e ao sistema de saúde, além de razões ou crenças pessoais que dificultam a adesão a tal prática ${ }^{(8)}$.

No entanto, estudos realizados com mulheres que passaram por essa experiência, descrevem-na como benéfica, pois possui efeito positivo, tanto no que se refere ao âmbito físico quanto ao emocional ${ }^{(2-3,5-5-6)}$.

Além disso, existe uma série de fatores motivadores para a adesão a um programa de reabilitação ou à prática de atividade física que são citados na literatura e muitas vezes apontados pelas próprias pacientes, destacando-se, dentre eles, a presença de profissionais especializados em reabilitação, grupos de apoio, suporte de familiares e reforço das orientações por meio de material ilustrativo ${ }^{(5-6-8,8,12-16)}$.

No que se refere às pacientes deste estudo, houve a preocupação de que as orientações a elas ministradas e a seus familiares fossem reforçadas por meio das ilustrações contidas no manual elaborado especificamente para esse fim. As pacientes referiram que o manual representou um importante recurso para o seguimento do programa proposto, pois as figuras e as explicações nele contidas eram claras, de fácil compreensão e auxiliavam-nas na realização dos exercícios no domicílio, longe dos olhares de um profissional da área da saúde.

Quanto ao apoio familiar, a literatura aponta esse componente como um dos fatores mais importantes para a adesão a qualquer tipo de tratamento, pois é na família que as pacientes encontram motivação, incentivo, suporte emocional e financeiro ${ }^{(5-6,12)}$. Por compreender os benefícios da participação da família em todo o processo de recuperação, as pesquisadoras do presente estudo tiveram o cuidado de propiciar a participação desta nos diferentes momentos em que as pacientes receberam as orientações. Acredita-se que o incentivo da família foi fundamental para que das 18 pacientes que aderiram ao programa, $13(72,2 \%)$ o tivessem feito, mesmo sentindo dificuldades. Por outro lado, a adesão ao programa por esse grupo de pacientes pode ser explicada com base no Modelo de Crenças em Saúde, pois as pacientes atribuíram ao programa de reabilitação um significado benéfico para a recuperação de sua saúde ${ }^{(11)}$.

Entretanto, houve fatores limitantes que restringiram a possibilidade de atender integralmente às necessidades dessas pacientes. Dentre eles, a falta de um programa formal de reabilitação de pacientes submetidas à cirurgia oncológica de mama na instituição onde o estudo foi realizado, bem como de estrutura física e de equipe multiprofissional especializada em reabilitação.

No que se refere à falta de vontade percebida por mulheres submetidas à cirurgia oncológica de mama, que tem sido apontada na literatura como a principal barreira à execução rotineira de atividade física ${ }^{(5)}$, no presente estudo, apenas duas pacientes $(7,1 \%)$ relataram esta dificuldade. Este fato talvez possa ser explicado porque o período estudado foi curto, restringindo-se ao tempo decorrido entre a alta hospitalar e o primeiro retorno ambulatorial. Nesse período, em geral, as principais preocupações das pacientes e dos familiares estão voltadas para a recuperação pós-operatória, fazendo com que todos estejam atentos ao cumprimento das orientações dos profissionais de saúde que visem atingir esse resultado.

Outro aspecto levantado foi o que se refere à dor após a cirurgia oncológica de mama. Estudos mostram que mais de $55 \%$ das pacientes submetidas a esse tipo de intervenção são acometidas por dor no pós-operatório que pode durar meses ou anos ${ }^{(17-18)}$. As sensações dolorosas, bem como as manifestações de formigamento, queimadura ou ardência, picada e irritação, referidas pelas mulheres após a cirurgia, pode estar relacionada à lesão do nervo intercostobraquial que é particularmente responsável pela alteração da sensibilidade dolorosa da região braquial proximalmedial e axilar ${ }^{(19)}$.

O Instituto Nacional do Câncer propõe algumas condutas para minimização do quadro álgico, tais como: cinesioterapia ativo-assistida, alongamento global, relaxamento cervical, automassagem linfática, deslocamento cicatricial e orientações específicas ${ }^{(20)}$.

As autoras deste estudo julgam que uma das orientações específicas diz respeito ao reforço da importância do uso sistemático de medicação antiálgica e, na falta de condições financeiras das pacientes para adquiri-la, se faz necessário que a instituição provenha os meios para que não falte este recurso, a fim de garantir melhor controle da dor pós-operatória.

Como algumas pacientes referiram ter sentido dor, tanto ao realizar os exercícios quanto em repouso, acredita-se que o uso de analgésicos, neste período, 
poderia aumentar a adesão ao programa de reabilitação, alcançando os resultados esperados e permitindo que as mesmas retomem suas atividades da vida diária, mais precocemente.

Chama a atenção o fato de que do total de pacientes que referiram dificuldades para a realização dos exercícios, 13 haviam sido submetidas a esvaziamento ganglionar axilar e 10 não haviam realizado esse procedimento. Esse dado sugere que o esvaziamento ganglionar axilar pode ter representado um dos fatores que dificultou a adesão das pacientes ao programa, devido à dor.

O esvaziamento ganglionar axilar durante a cirurgia de mama pode levar a complicações relacionadas à lesão de nervos, dentre os quais o intercostobraquial, que conforme mencionado anteriormente, responde pela alteração da sensibilidade dolorosa da região braquial proximal-medial e axilar ${ }^{(19)}$.

Cabe ressaltar, que as pacientes eram orientadas para que a amplitude do movimento durante a execução dos exercícios fosse restrita, até o ponto que não lhes causasse desconforto pela dor. Esse fato foi importante, pois deu à mulher a possibilidade de respeitar seu corpo, dandolhe a liberdade de conhecer seus novos limites para poder realizar os exercícios preconizados ${ }^{(5)}$.

Nove pacientes mencionaram que suas famílias, apreensivas, não permitiram que elas realizassem atividades domésticas, mesmo quando elas manifestaram vontade de realizá-las e sentiram que estavam em condições físicas para tal. Com isso, as pacientes referiram que embora reconhecessem o zelo da família, a inatividade fazia com que se sentissem inúteis ou inválidas, o que aponta a necessidade de orientações sobre quais atividades de vida diária a paciente pode realizar no pós-operatório ${ }^{(5,13)}$.

Sabe-se que o trabalho doméstico deve ser realizado moderadamente, pois a execução do mesmo é considerada fator de risco para o aparecimento de linfedema no braço homolateral à cirurgia, se realizado de maneira excessiva ${ }^{(13)}$.

Cabe destacar que na entrevista pré-operatória uma indagação freqüente das pacientes era se o braço iria voltar ao normal e se elas poderiam voltar a realizar os afazeres domésticos como antes. Considera-se importante o reforço das orientações às pacientes e familiares quanto a reassumir suas atividades de vida diária paulatinamente, a partir da alta hospitalar, desde que se sintam aptas e com disposição para tal.

A adoção dessas medidas poderá contribuir para a redução do medo, freqüente nessas pacientes, bem como propiciar a sua reintegração ao contexto social e familiar, o mais precocemente possível.

\section{CONCLUSÃO}

Os resultados deste estudo mostraram que a maioria das pacientes aderiu ao programa de exercícios para reabilitação do ombro homolateral à cirurgia. Mesmo assim, acredita-se que a taxa de adesão poderia ser maior se algumas medidas fossem implementadas, tais como, o melhor controle da dor pós-operatória por meio de terapia antiálgica adequada e reforço das orientações, a fim de que o medo da dor ao realizar os exercícios, ou de que estes possam ocasionar-lhes algum dano, possa ser minimizado.

Além disso, conforme apontado na literatura, o envolvimento da família no processo de reabilitação, bem como a condução desse processo por meio de um trabalho em grupo com pacientes que estão vivenciando a mesma experiência, poderia promover maior adesão ao programa.

\section{REFERÊNCIAS}

1. Camargo MC, Marx AG. Reabilitação física no câncer de mama. São Paulo: Roca; 2000.

2. Sasaki T, Lamari NM. Reabilitação funcional precoce pós mastectomia. HB Cient. 1997; 4(2): 121-7.

3. Pinto BM, Maruyama NC. Exercise in the rehabilitation of breast cancer survivors. Psychooncology. 1999; 8(3): 191-206.

4. Bendz I, Fagevik Olsén M. Evaluation of immediate versus delayed shoulder exercises after breast cancer surgery including lymph node dissection - a randomised controlled trial. Breast. 2002;11(3):241-8.

5. Prado MAS, Mamede MV, Almeida AM, Clapis MJ. A prática de atividade física em mulheres submetidas à cirurgia por câncer de mama: percepção de barreiras e benefícios. Rev Latinoam Enfermagem. 2004; 12(3):494-502.

6. Sales CAC, Paiva L, Scandiuzzi D, Anjos ACY. Qualidade de vida de mulheres tratadas de câncer de mama: funcionamento social. Rev Bras Cancerol. 2001;47(3):263-72.

7. Last JM. A dictionary of epidemiology. 4th ed. New York: Oxford University; 2001.

8. World Health Organization. Adherence to long-term therapies: Policy for Action. Meeting Report 4-5 June 2001. Geneva: WHO/MNC/CCH/01.02; 2001.

9. Kurita GP, Pimenta CAM. Adesão ao tratamento da dor crônica: estudo de variáveis demográficas, terapêuticas e psicossociais. Arq Neuropsiquiatr. 2003; 61(2-B): 416-25.

10. Moscati IM, Persano S, Castro LLC. Aspectos metodológicos e comportamentais da adesão à terapêutica. In: Castro LLC. Fundamentos da farmacoepidemiologia. São Paulo: AG; 2000. p. $171-80$.

11. Lescura Y, Mamede MV. Educação em saúde: abordagem para o enfermeiro. São Paulo: Sarvier; 1990.

12. Bergamasco RB, Angelo M. O sofrimento de descobrir-se com câncer de mama: como o diagnóstico é experienciado pela mulher. Rev Bras Cancerol. 2001;47(3):277-82.

13. Rodrigues DP, Melo EM, Silva RM, Mamede MV. O suporte social para atender as necessidades de mulheres mastectomizadas. Rev Bras Cancerol. 1998;44(3):231-8.

14. Bittencourt JFV, Cadete MMM. Vivências da mulher a ser mastectomizada: esclarecimentos e orientações. Rev Bras Enfermagem. 2002;55(4):420-3.

15. Gutiérrez MGR, Pinelli FGS, Areias VL, Barbi T Avaliação de um programa educativo sobre os cuidados com o sistema 
de drenagem para cuidadores de mulheres mastectomizadas. Acta Paul Enfermermagem. 2004;17(4):412-8.

16. Box RC, Reul-Hirche HM, Bullock-Saxton JE, Furnival CM. Shoulder movement after breast cancer surgery: results of a randomised controlled study of postoperative physiotherapy. Breast Cancer Res Treat. 2002; 75(1): 35-50.

17. Kwekkeboom K. Postmastectomy pain syndromes. Cancer Nurs.1996; 19(1):37-43.

18. Randal J. Post-mastectomy pain found to be common: treatment options sparse, but growing. J Natl Cancer Inst. 1998; 90(10):731-2.

19. Piato $S$, Piato JRM. Atenção fisioterapêutica após cirurgia para o câncer de mama. In: Piato S, Piato JRM. Doenças da mama. Rio de Janeiro: Revinter; 2006. p. 230-5.

20. Bergmann A, Ribeiro MJP, Pedrosa E, Nogueira EA, Oliveira ACG. Fisioterapia em mastologia oncológica: rotinas do Hospital do Câncer III / INCA. Rev Bras Cancerol. 2006;52(1):97-109.

\section{ANEXO}

Ficha de seguimento do programa de reabilitação

\section{Dados Pessoais}

Nome:

Contato:

Procedente de:
Idade: Estado civil: Escolaridade:
Cor:

R.H.

\section{II.Dados Clínicos}

Diagnóstico definitivo:

Data da cirurgia:

Tratamento: ( )Quadrantectomia ( ) Esvaziamento axilar ( )Radioterapia

( ) Mastectomia ( )Quimioterapia

III.Avaliação da realização dos exercícios no domicílio

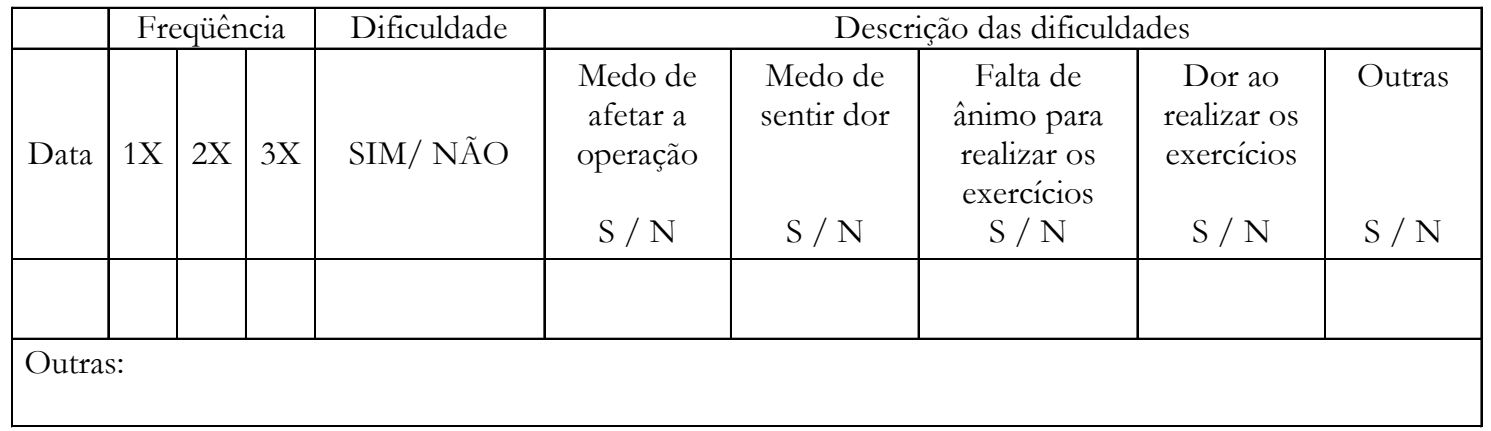

IV.Avaliação das atividades de vida diária nos retornos ambulatoriais

Usar a seguinte legenda:

- Não realizou a atividade: NR

- Realizou a atividade sem dificuldade: SD

- Realizou a atividade com pouca dificuldade: PD

- Realizou a atividade sem muita nem pouca dificuldade: +/-

- Realizou a atividade com muita dificuldade: MD

- Não conseguiu realizar a atividade: NC

\begin{tabular}{|l|l|l|}
\hline \multicolumn{1}{|c|}{ Atividades } & $\begin{array}{c}\text { Controle } \\
\text { pré- } \\
\text { operatório }\end{array}$ & $\begin{array}{c}\mathbf{1}^{\circ} \\
\text { retorno } \\
\text { amb. }\end{array}$ \\
\hline Tomar banho & & \\
\hline Lavar a parte superior do ombro oposto & & \\
\hline Escovar os dentes & & \\
\hline Pentear e/ou prender o cabelo & & \\
\hline Vestir-se e despir-se & & \\
\hline Alimentar-se sozinha & & \\
\hline Passar roupa & & \\
\hline Varrer a casa & & \\
\hline Arrumar armários & & \\
\hline Cozinhar & & \\
\hline Lavar louça & & \\
\hline Lavar roupa & & \\
\hline Dependurar roupa no varal & & \\
\hline Tirar pó dos móveis & & \\
\hline Arrumar a cama & & \\
\hline
\end{tabular}

